\title{
Understanding PTSD and Depression During the Pandemic Watch
}

\author{
Kristi Kanel* \\ Professor, Department of Human Services California State University, Fullerton, CA 92831, USA
}

*Corresponding author: Kristi Kanel, Professor, Department of Human Services California State University, Fullerton, CA 92831, USA

\begin{abstract}
The Covid 19 Pandemic has affected everyone worldwide. It has been especially challenging for the United States due to politicization of how to best manage it. For the past eight months, the USA, along with the rest of the world has been on Pandemic Watch. This wording is used to replace terms such as "stay at home", "quarantine', and "lockdown" since currently none of those things are really being enforced in the USA, although some countries do actually lockdown individuals, and some people who may be infected are asked to quarantine for two weeks. The focus of this paper is to develop an understanding of the psychological, emotional, behavioral, and social effects of being on Pandemic Watch has had on people, with a particular focus on US citizens. It will be suggested that Pandemic Watch is a better suited name since it most of us have been in an ongoing state of watching, waiting and being hypervigilant. This article will attempt to connect this state of Pandemic Watch to the depression and Post Traumatic Stress Disorder (PTSD) being experienced, primarily due to unknown end to the pandemic, not knowing how one will react if they get infected, not knowing if one will get infected, loss of freedom, loss of significant other, loss of jobs, and loss of normal life style. Additionally, the added stressors of the Presidential election, political divisiveness and racial unrest will be discussed as to how they also affect individuals during the pandemic.
\end{abstract}

Keywords: PTSD; Depression; Pandemic; Grief; Loss; Hypervigilance; Racial Unrest

\section{Introduction}

Since the world became familiar with the Pandemic related to the Novel Corona Virus, or COVID 19, most people have experienced some form of emotional, psychological, behavioral, or interpersonal changes. This is in large part due to the various levels of governing bodies setting certain boundaries related to social gatherings and congregating at work. The government and media used terms such as "stay at home", "self-quarantine", and "lockdown" to identify behaviors that encourage people to be minimize contact with others to remain safe. These terms may be perceived as setting boundaries on one's normal activities and social and occupational functioning. Certain restrictions and guidelines were set up by the Center for Disease Control in an effort to contain and minimize the spread of the virus such as wearing facial coverings, maintaining social distance of six feet, and avoiding social gatherings. These restrictions affected most people in that their normal way of living was significantly altered in a matter of 1-2 weeks. Adjustments had to be made quickly without much psychological preparation.
When people struggle to adjust to stressors, they may enter into crisis states [1-3]. This crisis state may manifest itself with a variety of symptoms leading to several diagnoses. The position of this paper is that this "Pandemic Watch" that has been in existence for over eight months as of this writing, has been an incubator for certain syndromes listed in the Diagnostic and Statistical Manual 5 such as Post Traumatic Stress Disorder, Acute Stress Disorder, Adjustment Disorders, and Generalized Anxiety disorder to name a few [4]. The term Pandemic Watch will be offered as a substitute term rather than "stay at home orders", quarantine" or "lock down" because those terms do not accurately reflect what has actually been the case in the USA.

\section{Theoretical Frameworks for Understanding Responses to the Pandemic Watch}

One might approach an understanding of how the Pandemic Watch phenomenon has led to various psychological states by 
looking at various theoretical positions. From the preventive psychiatry model laid forth by [2],Pandemic Watch can be thought of as "an obstacle that is, for a time, insurmountable by the use of customary methods of problem solving where a period of disorganization ensues, a period of upset, during which many abortive attempts to a solution are made", which is how Caplan described a crisis. Of course, those individuals that were able to manage all of the changes probably didn't go through a crisis state, or if they did, it was brief, and they may have sought help. This ability to invoke help has been described as a characteristic of effective coping by Caplan (1964) [5]. Those who were able to do this, according to Caplan, would have fared better than those that did not.

Other factors that may have contributed to whether someone prevented themselves from suffering from PTSD, depression, or anxiety disorders has to do with whether the person was able to gather accurate information, look at things realistically, express feelings, pacing themselves and being aware of fatigue, and having trust and optimism (Caplan, 1964) [5].

Gabbard's model (2000) [6] looks at causes of PTSD and other syndromes found in the DSM 5 from a psychodynamic psychiatric perspective. He states that "the most common precipitating event reported among persons with PTSD was the sudden, unexpected death of a loved one" (p. 252). He further suggests that trauma victims alternate between denying the event and compulsively repeating it as the mind's attempt to process and organize overwhelming stimuli. One could easily see how many people might have lose due to the pandemic while others might be waiting and watching for losses to occur. This sets up a well- known symptom of PTSD-hypervigilance. This refers to being alert, aroused, and ready. This state can affect not only psychological states but physiological states as well by triggering the autonomic nervous system, in particular increasing the sympathetic nervous system.

Other symptoms of PTSD that may be observed during the Pandemic Watch are depersonalization and derealization and numbing, which fall under the dissociative symptom response to trauma [4]. Individuals may feel disconnected from others and lose a sense of reality and a sense of who they are due to being isolated for many months. Numbing may also be seen in many as they interact with others via Zoom and other social media rather than in person. Many people talk about Zoom fatigue, (which may be similar to combat fatigue). When people are fatigued and feel powerless, they often just give up and exist just to survive rather than escape the situation [3]. This leads to a sense of being numb which actually might be helpful in relaxing the anxiety for a while. Overtime though, it may lead to a state often called "learned helplessness". This in turn may lead to depression. One of the symptoms of depression is feeling helpless.
When looking at depression from Kubler-Ross's (1969) [7] paradigm, it is a normal part of loss, the stage just prior to acceptance of the loss. Many people have suffered loss during the pandemic watch period. These losses include loss of loved ones to be sure. However, there have been many other types of loss, such as loss of social activities, loss of friends, loss of work and school, and a general loss of a way of life with freedom to choose how to manage daily activities. Because the pandemic has been continuous, it's been challenging for people to move into acceptance and move forward. The state of perpetual loss with no end in sight maintains a certain level of ongoing loss, though to the extent people can find new things to put energy into and withdraw from lost activities and significant others, they should be able to come to terms with their grief thereby reducing depression [8].

The Mental Health Center of North Iowa, Inc. (2005) [9] offers another model for understanding the psychological reactions to the Pandemic Watch. They discuss four phases of community disaster as being the heroic, the honeymoon, disillusionment, and reconstruction. If we plug in specifics from the pandemic period, we can propose that the heroic phase occurred from March through April when thousands died. The community did its part to help out by making masks, collecting PPE for health care workers, calling health care workers heroes, and during this time people thought it was temporary and were just living to stay healthy and existed in denial to some extent, or grief if loved one passed. Soon after April, the honeymoon period began. People felt better because the Center for Disease Control told us that wearing masks, social distancing, and washing hands would help prevent spread. Many of us thought life would resume to normal by summer when the weather changed. There was good feelings, albeit a tad unrealistic. Once the community realized that things were not going back to normal any time soon, not until there was a vaccine, many became angry and disillusioned. The stress of constant hypervigilance clinched PTSD for many, and anger is a symptom of PTSD not often talked about. However, we know that many of the most recent military service members returning from many deployments often experience anger as their primary negative mood when they suffer from PTSD [10]. The DSM 5 category of negative mood does not specify the type of negative mood, thereby making anger a potential symptom of PTSD as well as a normal part of the stages of loss. The final phase, Reconstruction, is an ongoing process at this point. The development of therapeutics to help COVID 19 infections is part of this phase as are the research studies trying to find a suitable vaccine for the world. Opening up businesses slowly is also part of this phase, and it looks like reconstruction will take at least another year to complete if we are lucky. It's possible that the fact that the majority of Americans voted for Joe Biden rather than the keeping with the current President, Donald Trump, was an attempt at reconstruction as well. 


\section{Discussion}

In light of these theoretical paradigms, it is not difficult to understand why many people in the community would be suffering from PTSD, Depression and anxiety. Not only has the community been forced to adjust to a new way of life due to Pandemic Watch, but they have also had to live through serious racial unrest and a very divided nation during 2020. Having multiple stressors to adjust to at the same time most certainly depletes our coping devices. Because of the racial unrest and political division, many have lost friendships and family relationships adding to the grief and loss from the pandemic. This may be the equivalent to the incubator for PTSD that many soldiers faced in Iraq and Afghanistan when experiencing multiple deployments [11].

With a potential new Federal level administration coming into power next year, it is possible that the United States community will move toward reconstruction and acceptance, guiding the process to reduce PTSD and depression in people. Certainly, a vaccine will help if it allows life to go back to normal. But we still do not know. So we still are in Pandemic Watch.
2. Caplan G (1961) An approach to community mental health. Grune \& Stratton, New York, NY.

3. Kanel K (2018) A guide to crisis intervention (6th ed.). Brooks/Cole, Pacific Grove, CA, USA.

4. American Psychiatric Association (2013) Diagnostic and statistical manual of mental disorders, Fifth edition. American Psychiatric Association publishing, Washington, DC.

5. Caplan G (1964) Principles of preventive psychiatry. Basic Books, New York, NY.

6. Gabbard GO (2000) Psychodynamic psychiatry in clinical practice (3rd ed.). American Psychiatric Press Inc, Washington, CD.

7. Kubler-Ross E (1969) On death and dying. Macmillan, New York, NY.

8. Worden WJ (1982) Grief counseling and grief therapy. Tavistock, London.

9. Mental Health Center of North Iowa, Inc (2005) Background Phases of Disaster. Author.

10. Jakupeak M, Conybeare D, Phelps L, Hunt S, Holmes HA, et al. (2007) Anger, hostility, and aggression among Iraq and Afghanistan war veterans reporting PTSD and subthreshold PTSD. Journal of Traumatic Stress 20(6): 945-954.

11. Yarvis J S, Schiess L (2008) Subthreshold PTSD as a predictor of depression, alcohol use, and health problems in soldiers. Journal of Workplace Behavioral health 23(4): 395-424.

\section{References}

1. James RK, Gilliland B E (2012) Crisis intervention strategies ( $7^{\text {th }}$ ed.) Brooks/Cole, Pacific Grove, CA, USA.

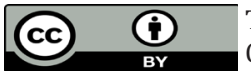

This work is licensed under Creative Commons Attribution 4.0 License

To Submit Your Article Click Here: Submit Article

DOI: $10.32474 / 0 J N B D .2020 .04 .000199$

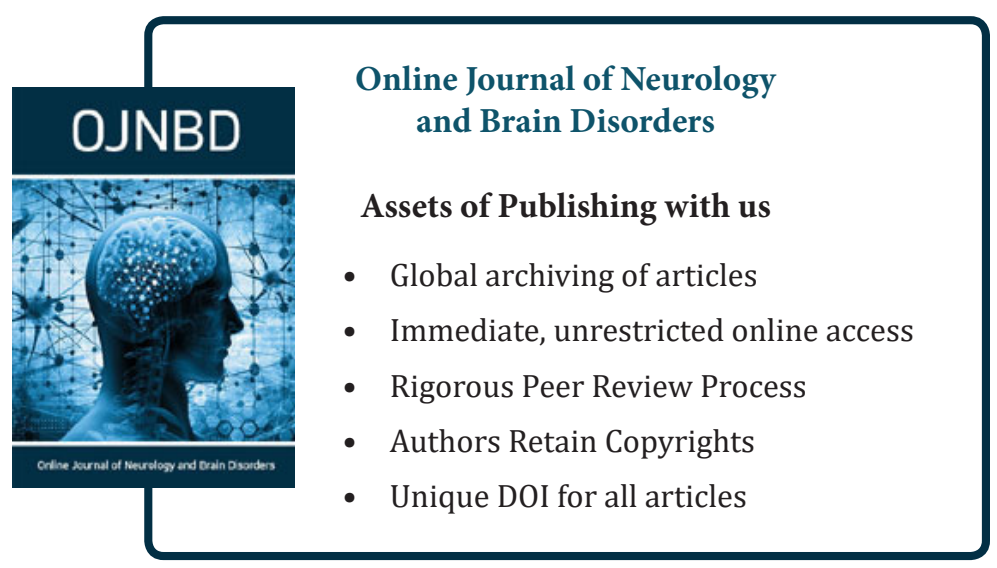

